\title{
HUMAN STATURE AND DIMENSIONS OF HAND AND FOOT- A SYSTEMATIC REVIEW
}

\author{
Charmode Sundip Hemant ${ }^{1}$, Kadlimatti Huchchesha Shivappa², Kuppast Nagesh ${ }^{3}$
}

${ }^{1}$ Asssistant Professor, Department of Anatomy, ESIC Medical College, Gulbarga, Karnataka, India.

2Professor and HOD, Department of Anatomy, ESIC Medical College, Gulbarga, Karnataka, India.

${ }^{3}$ Assistant Professor, Department of Forensic Medicine, ESIC Medical College, Gulbarga, Karnataka, India.

\section{ABSTRACT}

\section{BACKGROUND}

Estimation of human stature is one of the important aspects of any forensic investigation. Anatomists, forensic experts and anthropologists all over the world have studied dimensions of various parts of body and correlated them with stature. India is studded with many places of pilgrimage, and other religious places where people tend to walk bare footed for religious reasons. Various studies have been conducted in Indian population on the same subject in past. The principal goal of these studies was to create a database and to derive coefficient and linear regression coefficient for stature and hand and foot dimensions. The present study aims to systematically review all the observations achieved from previous studies done in India.

\section{METHODS}

A detailed online search of articles was made on various databases like MedLine, PubMed, Wiley Online Library, Science Direct, Google Scholar, Cochrane Library, Embase etc. from 2000 to present and 23 studies were found. Amongst these 23 studies, 15 studies were shortlisted based on their similarity as per study design, sample size, variables studied taking authors own study as reference study.

\section{RESULTS}

All the 23 shortlisted studies were reviewed, and observations were tabulated, statistically analysed and graphically represented.

\section{CONCLUSIONS}

Significant positive correlation was observed between human stature and hand and foot dimensions. Foot length was stated as a better predictor of stature compared to other dimensions. Human stature of north Indian population is higher than south Indian population. Hand and foot dimensions are higher in north Indian males than south Indian males.

HOW TO CITE THIS ARTICLE: Hemant CS, Shivappa KH, Nagesh K. Human stature and dimensions of hand and foot- a systematic review. J. Evolution Med. Dent. Sci. 2019;8(18):1503-1511, DOI: 10.14260/jemds/2019/334

\section{BACKGROUND}

Estimation of human stature is one of the important aspects of any forensic investigation. Anatomists, forensic experts and anthropologists all over the world have studied dimensions of various parts of body and correlated them with human stature and vice versa. India is studded with many pilgrimages and religious places and walking bare footed is a very common practice. Hence information about hand and foot dimensions and its correlation with human stature is of forensic importance. Various studies have been conducted in Indian population on the same subject in past. Unfortunately, most of those studies have yielded statistically insignificant conclusions. Also, a lot of diversity has been observed in the conclusions of similar studies done in same population of same region.

'Financial or Other Competing Interest': None.

Submission 18-07-2018, Peer Review 20-04-2019,

Acceptance 26-04-2019, Published 06-05-2019.

Corresponding Author:

Charmode Sundip Hemant,

Department of Anatomy,

ESIC Medical College,

Gulbarga,

Karnataka,

India.

E-mail: sundip.charmode@yahoo.com

DOI: $10.14260 /$ jemds $/ 2019 / 332$

\section{(c) $(1) \ominus$}

The principal goal of these studies was to study the correlation of dimensions of body parts with human stature and thereby to create a database of this population. They also derived a correlation coefficient and linear regression coefficient between stature and dimensions of different body parts.

The present review aims to systematically analyse the observations achieved from all previous similar studies done in Indian population. Focus was on studies on correlation of stature with hand and foot dimensions. It is believed that this systematic analysis will be useful for forensic investigations and anthropological studies conducted in Indian population.

\section{METHODS}

A detailed online search of articles was made on various databases like MedLine, PubMed, Wiley online library, science direct, google scholar, Cochrane library, Embase etc. from 2000 till present. Studies conducted by various authors on Indian population were searched (Either full pdf copy or abstract) and 22 such studies were found on correlation of human stature and hand and foot dimensions (Table 1). These studies varied in study design, age groups, variables studied and also in methodology used for taking measurements of hand and foot. 14 studies were shortlisted based on inclusion criteria (Table 2, Table 3). Observations were tabulated and analysed. 


\begin{tabular}{|c|c|c|c|c|c|}
\hline Sl. No. & Name of Author & Year of Study & Study Age Group (Yrs.) & Variables Studied & Place of Study \\
\hline 1 & Oommen et al ${ }^{1}$ & 2005 & $19-25$ & HL, FL & Mangalore \\
\hline 2 & Danborno and Elukpo ${ }^{2}$ & 2007 & M - 24.5, 22.2 & H, HL, HB, FL, FB & Nigeria \\
\hline 3 & Patel et $\mathrm{al}^{3}$ & 2007 & $17-22$ & $\mathrm{H}, \mathrm{FL}$ & Gujarat \\
\hline 4 & Krishnan $\mathrm{K}$ et al ${ }^{4}$ & 2007 & $17-20$ & H, HL, HB, FL, FB & Himachal Pradesh \\
\hline 5 & Rastogi et al 5 & 2008 & $20-30$ & $\mathrm{HL}, \mathrm{HB}, \mathrm{H}$ & Manipal \\
\hline 6 & Sen J et $\mathrm{al}^{6}$ & 2008 & $18-50$ & $\mathrm{H}, \mathrm{FL}, \mathrm{FB}$ & North Bengal \\
\hline 7 & Kanchan et al7 & 2008 & NM & $\mathrm{H}, \mathrm{FL}, \mathrm{FB}$ & North India \\
\hline 8 & Chikhalkar et al8 & 2009 & $19-23$ & H, W, FAL, HL, HB, FL, FB & Mumbai \\
\hline 9 & Krishan, et $\mathrm{al}^{9}$ & 2011 & $17-20$ & HL, HB, FL, FB & Chandigarh \\
\hline 10 & Patel, et al ${ }^{10}$ & 2012 & $17-23$ & $\mathrm{H}, \mathrm{FL}, \mathrm{FB}, \mathrm{HL}, \mathrm{HB}, \mathrm{AS}$ & Gujarat \\
\hline 11 & Ibegbu, et al 11 & 2013 & $5-10$ & $\mathrm{H}, \mathrm{HL}$ & Nigeria \\
\hline 12 & Mohite, et al ${ }^{12}$ & 2015 & $17-25$ & H, HL, HB, FL & Wardha \\
\hline 13 & Bodorikova and Nescakova ${ }^{13}$ & 2015 & $18-24$ & $\mathrm{H}, \mathrm{HL}, \mathrm{HB}, \mathrm{FL}, \mathrm{FB}$ & Slovakia \\
\hline 14 & Kavyashree et al ${ }^{14}$ & 2015 & $20-22$ & $\mathrm{H}, \mathrm{HL}, \mathrm{HB}$ & Karnataka \\
\hline 15 & Dey and Kapoor 15 & 2015 & Above 18 & HL, HB & New Delhi \\
\hline 16 & Pal, et al ${ }^{16}$ & 2016 & $20-40$ & HL, HB, W, H, DL & West Bengal \\
\hline 17 & Tandon et al 17 & 2016 & $18-30$ & $\mathrm{H}, \mathrm{HL}, \mathrm{HB}, \mathrm{FL}, \mathrm{FB}, \mathrm{DL}$ & Uttar Pradesh \\
\hline 18 & Dhaneria et al 18 & 2016 & $17-25$ & $\mathrm{H}, \mathrm{FL}, \mathrm{FB}$ & Rajasthan \\
\hline 19 & Shankar et al ${ }^{19}$ & 2017 & $18-25$ & $\mathrm{H}, \mathrm{HL}$ & Bangalore \\
\hline 20 & Kim, et al20 & 2018 & $20-59$ & $\mathrm{H}, \mathrm{HL}, \mathrm{HB}, \mathrm{FL}, \mathrm{FB}$ & Korea \\
\hline 21 & Samoon et al 21 & 2018 & $20-45$ & HL, $\mathrm{H}$ & Kashmir \\
\hline 22 & Ibrahim et al 22 & 2018 & $20-30$ & S, HL, PL, HB, FL & Saudi Arabia \\
\hline \multicolumn{6}{|c|}{ Table 1. List of Previous Studies } \\
\hline \multicolumn{6}{|c|}{$\begin{array}{l}\text { H- Height, HL - Hand length, HB - Hand breadth, FL - Foot length, FB - Foot breadth, } \\
\text { PL - Palm length, DL - Digit/ finger length, AS-Arm span, FAL - Forearm length, } \\
\text { NM - Not measured NC - Not comparable, NA - Data not available }\end{array}$} \\
\hline
\end{tabular}

\begin{tabular}{|c|c|c|c|c|c|}
\hline Sl. No. & Name of Author & Year of Study & $\begin{array}{c}\text { Study Age Group } \\
\text { (Yrs.) }\end{array}$ & Variables Studied & Place of Study \\
\hline 1 & Patel et al ${ }^{3}$ & 2007 & $17-22$ & $\mathrm{H}, \mathrm{FL}$ & Gujarat \\
\hline 2 & Krishnan $\mathrm{K}$ et al ${ }^{4}$ & 2007 & $17-20$ & $\mathrm{H}, \mathrm{HL}, \mathrm{HB}, \mathrm{FL}, \mathrm{FB}$ & Himachal Pradesh \\
\hline 3 & Rastogi et al ${ }^{5}$ & 2008 & $20-30$ & $\mathrm{HL}, \mathrm{HB}, \mathrm{H}$ & Manipal \\
\hline 4 & Sen J et $\mathrm{al}^{6}$ & 2008 & $18-50$ & H, FL, FB & North Bengal \\
\hline 5 & Kanchan et al7 & 2008 & NM & H, FL, FB & North India \\
\hline 6 & Chikhalkar et al ${ }^{8}$ & 2009 & $19-23$ & H, W, FAL, HL, HB, FL, FB & West Maharashtra \\
\hline 7 & Patel, et al ${ }^{10}$ & 2012 & $17-23$ & $\mathrm{H}, \mathrm{FL}, \mathrm{FB}, \mathrm{HL}, \mathrm{HB}, \mathrm{AS}$ & Gujarat \\
\hline 8 & Mohite, et al 12 & 2015 & $17-25$ & $\mathrm{H}, \mathrm{HL}, \mathrm{HB}, \mathrm{FL}$ & East Maharashtra \\
\hline 9 & Kavyashree et al ${ }^{14}$ & 2015 & $20-22$ & $\mathrm{H}, \mathrm{HL}, \mathrm{HB}$ & Karnataka \\
\hline 10 & Pal, et al 16 & 2016 & $20-40$ & HL, HB, W, H, DL & West Bengal \\
\hline 11 & Tandon et al ${ }^{17}$ & 2016 & $18-30$ & $\mathrm{H}, \mathrm{HL}, \mathrm{HB}, \mathrm{FL}, \mathrm{FB}, \mathrm{DL}$ & Uttar Pradesh \\
\hline 12 & Dhaneria et al ${ }^{18}$ & 2016 & $17-25$ & H, FL, FB & Rajasthan \\
\hline 13 & Shankar et al ${ }^{19}$ & 2017 & $18-25$ & $\mathrm{H}, \mathrm{HL}$ & Bangalore \\
\hline 14 & Samoon et al21 & 2018 & $20-45$ & $\mathrm{HL}, \mathrm{H}$ & Kashmir \\
\hline \multicolumn{6}{|c|}{ Table 2. Shortlisted Studies for Review } \\
\hline
\end{tabular}

\begin{tabular}{|c|c|c|}
\hline Sl. No. & Criteria for Shortlisting & No. of Studies \\
\hline 1. & Studies done on Indian population & 18 studies \\
\hline 2 & Age group of study population & 17 years and above \\
\hline 3 & $\begin{array}{l}\begin{array}{c}\text { Studies correlating stature with hand or foot } \\
\text { dimensions }\end{array} \\
\end{array}$ & 15 studies \\
\hline 4 & Measurement of human stature & The height was measured from the sole of the feet to the vertex of the head 11 \\
\hline 5 & Measurement of hand length & $\begin{array}{c}\text { Hand length (HL) was measured as the distance between the distal crease of } \\
\text { wrist to tip of middle finger }{ }^{12}\end{array}$ \\
\hline 6 & Measurement of hand breadth & $\begin{array}{l}\text { Hand Breadth (HB) was measured from } 1^{\text {st }} \text { metacarpophalangeal joint to } \\
\text { base of } 5^{\text {th }} \text { metacarpal12 }\end{array}$ \\
\hline 7 & Measurement of foot length & $\begin{array}{l}\text { Foot length (FL) was measured as the distance between the posterior most } \\
\text { point of the heel and the anterior most point of the foot }{ }^{1} \text {. }\end{array}$ \\
\hline 8 & Measurement of foot breadth & $\begin{array}{c}\text { Foot breadth (FB) was measured as distance between Metatarsal tibiale } \\
\text { (point projecting most medially on the head of the } 1^{\text {st }} \text { metatarsal bone) and } \\
\text { Metatarsal Fibulare (point projecting most laterally on the head of the } 5^{\text {th }} \\
\text { metatarsal bone) }{ }^{17}\end{array}$ \\
\hline \multicolumn{3}{|c|}{ Table 3. Inclusion Criteria for Shortlisting Studies } \\
\hline
\end{tabular}




\begin{tabular}{|c|c|c|c|c|c|c|c|}
\hline \multirow{2}{*}{$\begin{array}{l}\text { Sl. } \\
\text { No. }\end{array}$} & \multirow{2}{*}{$\begin{array}{l}\text { Name of } \\
\text { Study }\end{array}$} & \multirow{2}{*}{$\begin{array}{l}\text { Place of } \\
\text { Study }\end{array}$} & \multirow{2}{*}{$\begin{array}{l}\text { Subject of } \\
\text { Study }\end{array}$} & \multicolumn{4}{|c|}{ Methods of Measurement } \\
\hline & & & & HL & HB & FL & FB \\
\hline 1. & $\begin{array}{l}\text { Oommen } \\
\text { et al }{ }^{1}\end{array}$ & Indian & $\begin{array}{c}\text { Correlates HL and } \\
\text { FL }\end{array}$ & $\begin{array}{l}\text { Midpoint of inter- } \\
\text { styloid line to tip of } \\
\text { middle finger }\end{array}$ & NM & $\begin{array}{l}\text { Distance between the } \\
\text { posterior most point of } \\
\text { the heel and anterior } \\
\text { most point of the foot }\end{array}$ & NM \\
\hline 2 & $\begin{array}{c}\text { Danborno } \\
\text { and Elukpo }^{2}\end{array}$ & $\begin{array}{l}\text { Foreign } \\
\text { study }\end{array}$ & \begin{tabular}{|c|}
$\begin{array}{c}\text { Correlates stature } \\
\text { with hand and foot } \\
\text { dimensions }\end{array}$ \\
\end{tabular} & $\begin{array}{c}\text { As done by Krishnan } \\
\text { K et al }{ }^{4}\end{array}$ & $\begin{array}{c}\text { As done by } \\
\text { Krishnan K et al }{ }^{4}\end{array}$ & $\begin{array}{c}\text { As done by Krishnan } \mathrm{K} \\
\text { et } \mathrm{al}^{4}\end{array}$ & $\mid \begin{array}{c}\text { As done by } \\
\text { Krishnan K et al }{ }^{4}\end{array}$ \\
\hline 3 & Patel et $\mathrm{al}^{3}$ & Indian & $\begin{array}{c}\text { Correlates stature } \\
\text { with hand and foot } \\
\text { dimensions }\end{array}$ & NM & NM & \begin{tabular}{|} 
Distance between the \\
posterior most point of \\
the heel and anterior \\
most point of the foot
\end{tabular} & NM \\
\hline 4 & $\begin{array}{c}\text { Krishnan K } \\
\text { et al }{ }^{4}\end{array}$ & Indian & \begin{tabular}{|c|}
$\begin{array}{c}\text { Correlates stature } \\
\text { with hand and foot } \\
\text { dimensions }\end{array}$ \\
\end{tabular} & NA & NA & NA & NA \\
\hline 5 & $\begin{array}{l}\text { Rastogi } \\
\text { et } \mathrm{al}^{5}\end{array}$ & Indian & $\begin{array}{c}\text { Correlates stature } \\
\text { with hand and foot } \\
\text { dimensions }\end{array}$ & $\begin{array}{c}\text { HL -1: Distal wrist } \\
\text { crease to tip of } \\
\text { middle finger } \\
\text { HL-2: Midpoint of } \\
\text { inter-styloid line to } \\
\text { tip of middle finger }\end{array}$ & $\begin{array}{c}\text { Measured between } \\
\text { radial side of the } \\
\text { second metacarpo- } \\
\text { phalangeal joint } \\
\text { and ulnar side of } \\
\text { the fifth } \\
\text { metacarpo- } \\
\text { phalangeal joint }\end{array}$ & NM & NM \\
\hline 6 & $\begin{array}{l}\text { Sen J } \\
\text { et al } 6\end{array}$ & Indian & \begin{tabular}{|c|}
$\begin{array}{c}\text { Correlates stature } \\
\text { with hand and foot } \\
\text { dimensions }\end{array}$ \\
\end{tabular} & NA & NA & NA & NA \\
\hline 7 & $\begin{array}{l}\text { Kanchan } \\
\text { et al } 7\end{array}$ & Indian & \begin{tabular}{|c|}
$\begin{array}{c}\text { Correlates stature } \\
\text { with hand and foot } \\
\text { dimensions }\end{array}$ \\
\end{tabular} & NA & NA & NA & NA \\
\hline 8 & $\begin{array}{c}\text { Chikhalkar } \\
\text { et al }{ }^{8}\end{array}$ & Indian & $\begin{array}{c}\text { Correlates stature } \\
\text { with hand and foot } \\
\text { dimensions }\end{array}$ & \begin{tabular}{|c|} 
From mid-point \\
below radial \& ulnar \\
tuberosity to tip of \\
middle finger \\
\end{tabular} & $\begin{array}{c}\text { From base of fifth } \\
\text { to second } \\
\text { metacarpal } \\
\text { (position - prone) }\end{array}$ & $\begin{array}{l}\text { From tip of toe to heel } \\
\text { on the medial side }\end{array}$ & $\begin{array}{l}\text { From base of } \\
\text { first to fifth } \\
\text { metatarsal }\end{array}$ \\
\hline 9 & $\begin{array}{l}\text { Krishan } \\
\text { et al }{ }^{9}\end{array}$ & Indian & $\begin{array}{c}\text { Correlates Sex with } \\
\text { HL, HB, FL and FB }\end{array}$ & NA & NA & NA & NA \\
\hline 10 & $\begin{array}{l}\text { Patel, } \\
\text { et al }{ }^{10}\end{array}$ & Indian & $\begin{array}{c}\text { Correlates stature } \\
\text { with hand and foot } \\
\text { dimensions }\end{array}$ & $\begin{array}{l}\text { Midpoint of inter- } \\
\text { styloid line to tip of } \\
\text { middle finger }\end{array}$ & $\begin{array}{c}\text { Measured at level } \\
\text { of metacarpo- } \\
\text { phalangeal joint. }\end{array}$ & $\begin{array}{l}\text { Distance between } \\
\text { anterior \& posterior } \\
\text { point of foot }\end{array}$ & $\begin{array}{c}\text { Measured at } \\
\text { level of } \\
\text { metatarsophalan } \\
\text { geal joint. }\end{array}$ \\
\hline 11 & $\begin{array}{l}\text { Ibegbu, } \\
\text { et al11 }\end{array}$ & Indian & $5-10$ years & $\begin{array}{l}\text { Findings not } \\
\text { applicable }\end{array}$ & $\begin{array}{l}\text { Findings not } \\
\text { applicable }\end{array}$ & Findings not applicable & $\begin{array}{l}\text { Findings not } \\
\text { applicable }\end{array}$ \\
\hline 12 & $\begin{array}{l}\text { Mohite, } \\
\text { et al }{ }^{12}\end{array}$ & Indian & $\begin{array}{c}\text { Correlates stature } \\
\text { with hand and foot } \\
\text { dimensions }\end{array}$ & $\begin{array}{l}\text { Midpoint of inter- } \\
\text { styloid line to tip of } \\
\text { middle finger }\end{array}$ & \begin{tabular}{|c|} 
From first \\
metacarpo- \\
phalangeal joint to \\
base of fifth \\
metacarpal \\
\end{tabular} & $\begin{array}{l}\text { Distance between } \\
\text { anterior \& posterior } \\
\text { point of foot }\end{array}$ & NM \\
\hline 13 & $\begin{array}{c}\text { Bodorikova } \\
\text { and } \\
\text { Nescakova }^{13}\end{array}$ & Foreign & \begin{tabular}{|c|}
$\begin{array}{c}\text { Correlates stature } \\
\text { with hand and foot } \\
\text { dimensions }\end{array}$ \\
\end{tabular} & NA & NA & NA & NA \\
\hline 14 & $\begin{array}{c}\text { Kavyashree } \\
\text { et al }^{14}\end{array}$ & Indian & $\begin{array}{c}\text { Correlates stature } \\
\text { with hand and foot } \\
\text { dimensions }\end{array}$ & $\begin{array}{l}\text { Straight distance } \\
\text { between the } \\
\text { midpoint of a line } \\
\text { joining the two } \\
\text { styloid process of } \\
\text { radius and ulna and } \\
\text { the lowest point on } \\
\text { the anterior margin } \\
\text { of the middle finger. }\end{array}$ & \begin{tabular}{|} 
Straight distance \\
between the most \\
medially placed \\
point on the head \\
of second \\
metacarpal on the \\
stretched hand and \\
the most laterally \\
placed point on the \\
head of fifth \\
metacarpal on the \\
stretched hand. \\
\end{tabular} & NM & NM \\
\hline 15 & $\begin{array}{l}\text { Dey and } \\
\text { Kapoor } \\
\text { et al15 }\end{array}$ & Indian & $\begin{array}{c}\text { Correlates HL and } \\
\text { HB }\end{array}$ & \begin{tabular}{|c|} 
Mid-point of a line \\
joining the two \\
styloid process and \\
tip of the middle \\
finger.
\end{tabular} & $\begin{array}{c}\text { Distance between } \\
\text { metacarpal } \\
\text { radialis and } \\
\text { metacarpal ulnare. }\end{array}$ & NM & NM \\
\hline
\end{tabular}




\begin{tabular}{|c|c|c|c|c|c|c|c|}
\hline & & & & & & & \\
\hline 16 & Pal et al 16 & Indian & $\begin{array}{c}\text { Correlates stature } \\
\text { with hand and foot } \\
\text { dimensions }\end{array}$ & $\begin{array}{c}\text { Distal wrist crease } \\
\text { to tip of middle } \\
\text { finger }\end{array}$ & \begin{tabular}{|} 
Distance between \\
metacarpal \\
radialis and \\
metacarpal ulnare.
\end{tabular} & NM & NM \\
\hline 17 & $\begin{array}{l}\text { Tandon } \\
\text { et al }{ }^{17}\end{array}$ & Indian & $\begin{array}{c}\text { Correlates stature } \\
\text { with hand and foot } \\
\text { dimensions }\end{array}$ & $\begin{array}{l}\text { Tip of the styloid } \\
\text { process of the radius } \\
\text { to the tip of the } \\
\text { middle finger }\end{array}$ & $\begin{array}{c}\text { Distance between } \\
\text { the most } \\
\text { prominent point } \\
\text { outside of the } \\
\text { lower epiphysis of } \\
\text { second metacarpal } \\
\text { to the most } \\
\text { prominent inside } \\
\text { of the lower } \\
\text { epiphysis of fifth } \\
\text { metacarpal. }\end{array}$ & $\begin{array}{l}\text { Distance between } \\
\text { anterior \& posterior } \\
\text { point of foot }\end{array}$ & $\begin{array}{c}\text { Distance } \\
\text { between the } \\
\text { most medially } \\
\text { projecting point } \\
\text { on the head of } \\
\text { the first } \\
\text { metatarsal bone } \\
\text { and most } \\
\text { laterally } \\
\text { projecting point } \\
\text { on the head of } \\
\text { the fifth } \\
\text { metatarsal bone }\end{array}$ \\
\hline 18 & $\begin{array}{c}\text { Dhaneria } \\
\text { et al } 18\end{array}$ & Indian & $\begin{array}{c}\text { Correlates stature } \\
\text { with hand and foot } \\
\text { dimensions }\end{array}$ & NM & NM & $\begin{array}{l}\text { Distance between } \\
\text { anterior \& posterior } \\
\text { point of foot }\end{array}$ & $\begin{array}{c}\text { Distance } \\
\text { between the } \\
\text { most prominent } \\
\text { point on the } \\
\text { medial aspect of } \\
\text { head of first } \\
\text { metatarsal and } \\
\text { most prominent } \\
\text { point on lateral } \\
\text { aspect of the } \\
\text { head of fifth } \\
\text { metatarsal }\end{array}$ \\
\hline 19 & $\begin{array}{c}\text { Shankar } \\
\text { et al }{ }^{19}\end{array}$ & Indian & $\begin{array}{c}\text { Correlates stature } \\
\text { with hand and foot } \\
\text { dimensions }\end{array}$ & Not specified & NM & NM & NM \\
\hline 20 & Kim, et al20 & Foreign & $\begin{array}{c}\text { Correlates stature } \\
\text { with hand and foot } \\
\text { dimensions }\end{array}$ & NA & NA & NA & NA \\
\hline 21 & $\begin{array}{c}\text { Samoon } \\
\text { et } \mathrm{al}^{21}\end{array}$ & Indian & \begin{tabular}{|c|}
$\begin{array}{c}\text { Correlates stature } \\
\text { with hand and foot } \\
\text { dimensions }\end{array}$ \\
\end{tabular} & $\begin{array}{l}\text { The proximal crease } \\
\text { of the wrist to the } \\
\text { tip of middle finger. }\end{array}$ & NM & NM & NM \\
\hline 22 & $\begin{array}{c}\text { Ibrahim } \\
\text { et } \mathrm{al}^{22}\end{array}$ & Foreign & $\begin{array}{c}\text { Correlates stature } \\
\text { with hand and foot } \\
\text { dimensions }\end{array}$ & $\begin{array}{l}\text { Distal crease of the } \\
\text { wrist and the tip of } \\
\text { the middle finger }\end{array}$ & \begin{tabular}{|} 
Distance between \\
the most remote \\
points on the \\
heads of the first \\
and fifth \\
metacarpal bones
\end{tabular} & NM & NM \\
\hline \multicolumn{8}{|c|}{ Table 4. List of Studies with Methods of Measurement } \\
\hline \multicolumn{8}{|c|}{$\begin{array}{l}\text { H- Height, HL - Hand length, HB - Hand breadth, FL - Foot length, FB - Foot breadth, } \\
\text { PL - Palm length, DL - Digit/ finger length, AS-Arm span, FAL - Forearm length, } \\
\text { NM - Not measured NC - Not comparable, NA - Data not available }\end{array}$} \\
\hline
\end{tabular}

\section{RESULTS}

\begin{tabular}{|c|c|c|c|c|c|c|c|c|}
\hline \multirow{3}{*}{$\begin{array}{l}\text { Sl. } \\
\text { No. }\end{array}$} & \multirow{3}{*}{$\begin{array}{l}\text { Name of } \\
\text { Study }\end{array}$} & \multirow{3}{*}{$\begin{array}{l}\text { Sample } \\
\text { Size (n) }\end{array}$} & \multirow{3}{*}{$\begin{array}{c}\text { Parameters } \\
\text { Studied }\end{array}$} & \multicolumn{5}{|c|}{ Observations } \\
\hline & & & & \multirow{2}{*}{$\begin{array}{l}\text { Mean Height } \\
\text { (cm) M/F }\end{array}$} & \multicolumn{2}{|c|}{ Mean Hand Length (cm) } & \multicolumn{2}{|c|}{ Mean Hand Breadth (cm) } \\
\hline & & & & & Right M/F & Left $M / F$ & Right M/F & Left $M / F$ \\
\hline 1 & Oommen et al ${ }^{1}$ & 100 & HL, FL & NA & $19.06 / 17.32$ & $19.06 / 17.24$ & NM & NM \\
\hline 2 & $\begin{array}{c}\text { Danborno and } \\
\text { Elukpo }^{2}\end{array}$ & 400 & $\mathrm{H}, \mathrm{HL}, \mathrm{HB}, \mathrm{FL}, \mathrm{FB}$ & $173.7 / 160.0$ & $19.85 / 18.51$ & $19.93 / 18.52$ & $8.90 / 7.82$ & $8.68 / 7.72$ \\
\hline 3 & Patel et $\mathrm{al}^{3}$ & 502 & $\mathrm{H}, \mathrm{FL}$ & $170.96 / 156.14$ & NM & NM & NM & NM \\
\hline 4 & $\begin{array}{c}\text { Krishnan K et al } 4 \\
\text { (2007) }\end{array}$ & 246 & $\mathrm{H}, \mathrm{HL}, \mathrm{HB}, \mathrm{FL}, \mathrm{FB}$ & NA & NA & NA & NA & NA \\
\hline 5 & Rastogi et al 5 & 500 & $\mathrm{HL}, \mathrm{HB}, \mathrm{H}$ & NA & NA & NA & NA & NA \\
\hline 6 & Sen J et al 6 & 450 & H, FL, FB & $\begin{array}{l}\text { Data not } \\
\text { available }\end{array}$ & NM & NM & NM & NM \\
\hline 7 & $\begin{array}{l}\text { Kanchan et al } 7 \\
(2008)\end{array}$ & 200 & $\mathrm{H}, \mathrm{FL}, \mathrm{FB}$ & $\begin{array}{l}\text { Data not } \\
\text { available }\end{array}$ & NM & NM & NM & NM \\
\hline 8 & Chikhalkar et al 8 & 300 & H, W, FAL, HL, HB, & 167.26 & 18.93 & 18.93 & 7.53 & 7.53 \\
\hline
\end{tabular}




\begin{tabular}{|c|c|c|c|c|c|c|c|c|}
\hline & & & FL, FB & & & & & \\
\hline 9 & Krishan et al9 & 246 & HL, HB, FL, FB & NA & NA & NA & NA & NA \\
\hline 10 & $\begin{array}{l}\text { Patel et al }^{10} \\
(2012)\end{array}$ & 273 & $\begin{array}{c}\text { H, FL, FB, HL, HB, } \\
\text { AS }\end{array}$ & 164.59 & \multicolumn{2}{|c|}{17.75} & \multicolumn{2}{|c|}{7.91} \\
\hline 11 & Ibegbu, et al 11 & 600 children & $\mathrm{H}, \mathrm{HL}$ & $\mathrm{NC}$ & $\mathrm{NC}$ & $\mathrm{NC}$ & $\mathrm{NC}$ & $\mathrm{NC}$ \\
\hline 12 & Mohite, et al ${ }^{12}$ & 230 & $\mathrm{H}, \mathrm{HL}, \mathrm{HB}, \mathrm{FL}$ & $171.12 / 159.43$ & \multicolumn{2}{|c|}{ Male - 19.11, Female - 17.40} & \multicolumn{2}{|c|}{ Male - 86.45, female - 73.74} \\
\hline 13 & \begin{tabular}{|c|} 
Bodorikova and \\
Nescakova 13 \\
\end{tabular} & 250 & H, HL, HB, FL, FB & NA & NA & NA & NA & NA \\
\hline 14 & $\begin{array}{c}\text { Kavyashree et } \\
\text { al }^{14}\end{array}$ & 294 & $\mathrm{H}, \mathrm{HL}, \mathrm{HB}$ & $\begin{array}{c}S M-171.73 \\
N M-169.04 \\
S F-159.25 \\
N F-158.42 \\
\end{array}$ & 18.81 & 18.74 & 8.24 & 8.00 \\
\hline 15 & Dey and Kapoor 15 & 147 & $\mathrm{HL}, \mathrm{HB}$ & NM & $19.2 / 17.3$ & $19.0 / 16.5$ & $8.3 / 7.57$ & $8.18 / 7.45$ \\
\hline 16 & Pal, et al ${ }^{16}$ & $\begin{array}{c}1662 \\
\text { women }\end{array}$ & HL, HB, W, H, DL & NM & 16.3 & 16.31 & 7.05 & 7.03 \\
\hline 17 & Tandon et al ${ }^{17}$ & 497 & $\begin{array}{c}\text { H, HL, HB, FL, FB, } \\
\text { DL }\end{array}$ & $172.7 / 157.1$ & $19.3 / 17.3$ & NM & \multicolumn{2}{|c|}{ Male - 8.3, Female - 7.2} \\
\hline 18 & Dhaneria et al 18 & 500 & $\mathrm{H}, \mathrm{FL}, \mathrm{FB}$ & $166.43 / 160.91$ & NM & NM & NM & NM \\
\hline 19 & Shankar et al ${ }^{19}$ & 220 & $\mathrm{H}, \mathrm{HL}$ & $\begin{array}{c}\text { Data not } \\
\text { available }\end{array}$ & $18.21 / 18.81$ & $18.35 / 18.82$ & NM & NM \\
\hline 20 & Kim, et al 20 & 5195 & H, HL, HB, FL, FB & NA & NA & NA & NA & NA \\
\hline 21 & Samoon et al21 & 158 & $\mathrm{HL}, \mathrm{H}$ & NM & NM & NM & NM & NM \\
\hline 22 & Ibrahim et al 22 & 350 & S, HL, PL, HB, FL & $175.44 / 158.96$ & $20.11 / 18.65$ & $20.75 / 18.6$ & $8.76 / 7.66$ & $8.7 / 7.62$ \\
\hline
\end{tabular}

\section{Table 5. Studies with Observations of Height and Hand Dimensions}

H- Height, HL - Hand length, HB - Hand breadth, FL - Foot length, FB - Foot breadth, PL - Palm length, DL - Digit/ finger length, AS-Arm span, FAL - Forearm length, NM - Not measured NC - Not comparable, NA - Data not available, SM -South male, SF - South female, NM - North male, NF - North female.

\begin{tabular}{|c|c|c|c|c|c|c|c|c|}
\hline \multirow{3}{*}{$\begin{array}{l}\text { Sl. } \\
\text { No. }\end{array}$} & \multirow{3}{*}{ Name of Study } & \multirow{3}{*}{$\begin{array}{c}\text { Sample } \\
\text { Size }\end{array}$} & \multirow{3}{*}{$\begin{array}{l}\text { Parameters } \\
\text { Studied }\end{array}$} & \multicolumn{5}{|c|}{ Observations } \\
\hline & & & & \multirow{2}{*}{$\begin{array}{c}\text { Mean Height } \\
\mathbf{M} / \mathbf{F}\end{array}$} & \multicolumn{2}{|c|}{ Mean Foot Length } & \multicolumn{2}{|c|}{ Mean Foot Breadth } \\
\hline & & & & & Right M/F & Left M/F & Right M/F & Left M/F \\
\hline 1 & Anitha Oommen et al ${ }^{1}$ & 100 & HL, FL & NA & $26.21 / 23.75$ & $26.0 / 23.68$ & NM & $\mathrm{NM}$ \\
\hline 2 & $\begin{array}{c}\text { B Danborno, A Elukpo } \\
\text { et } \mathrm{al}^{2}\end{array}$ & 400 & H, HL, HB, FL, FB & $173.7 / 160.0$ & $28.39 / 24.52$ & $26.42 / 24.70$ & $9.02 / 8.23$ & $9.09 / 8.11$ \\
\hline 3 & $\begin{array}{c}\text { Patel SM, Shah GV et } \\
\mathrm{al}^{3}\end{array}$ & 502 & $\mathrm{H}, \mathrm{FL}$ & $170.96 / 156.14$ & Male - 24.44 & Female -22.34 & NM & NM \\
\hline 4 & $\begin{array}{c}\text { Krishnan } \mathrm{K} \text { et al } \\
\text { (2007) }\end{array}$ & 246 & H, HL, HB, FL, FB & NA & NA & NA & NA & NA \\
\hline 5 & 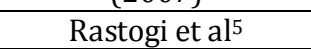 & 500 & HL, HB, H & NA & NM & NM & NM & NM \\
\hline 6 & Sen J et al 6 & 450 & H, FL, FB & $\begin{array}{l}\text { Data not } \\
\text { available }\end{array}$ & $\begin{array}{l}\text { Data not } \\
\text { available }\end{array}$ & $\begin{array}{l}\text { Data not } \\
\text { available }\end{array}$ & $\begin{array}{l}\text { Data not } \\
\text { available }\end{array}$ & $\begin{array}{l}\text { Data not } \\
\text { available }\end{array}$ \\
\hline 7 & Kanchan et al7 (2008) & 200 & $\mathrm{H}, \mathrm{FL}, \mathrm{FB}$ & $\begin{array}{l}\text { Data not } \\
\text { available }\end{array}$ & $\begin{array}{l}\text { Data not } \\
\text { available }\end{array}$ & $\begin{array}{l}\text { Data not } \\
\text { available }\end{array}$ & $\begin{array}{l}\text { Data not } \\
\text { available }\end{array}$ & $\begin{array}{l}\text { Data not } \\
\text { available }\end{array}$ \\
\hline 8 & $\begin{array}{c}\text { Chikhalkar BG et al }{ }^{8} \\
\text { (2008) }\end{array}$ & 300 & $\begin{array}{l}\text { H, W, FAL, HL, } \\
\text { HB, FL, FB }\end{array}$ & 167.26 & \multicolumn{2}{|c|}{24.008 with SD 1.420} & \multicolumn{2}{|c|}{8.895 with SD 0.703} \\
\hline 9 & 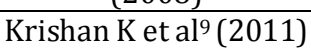 & 246 & HL, HB, FL, FB & NA & NA & NA & NA & NA \\
\hline 6 & $\begin{array}{c}\text { Patel PN, Tanna JA et } \\
\mathrm{al}^{10}(2012)\end{array}$ & 273 & $\begin{array}{c}\text { H, FL, FB, HL, HB, } \\
\text { AS }\end{array}$ & 164.59 & \multicolumn{2}{|c|}{24.178 with SD 1.809} & \multicolumn{2}{|c|}{9.28 with SD 0.865} \\
\hline 7 & $\begin{array}{l}\text { A. Ibegbu, David et } \\
\text { al }^{8}(2013)\end{array}$ & $\begin{array}{c}600 \\
\text { children }\end{array}$ & $\mathrm{H}, \mathrm{HL}$ & NC & $\mathrm{NC}$ & $\mathrm{NC}$ & NC & $\mathrm{NC}$ \\
\hline 8 & \begin{tabular}{|c|} 
Prakash M. Mohite et \\
$\mathrm{al}^{9}(2015)$ \\
\end{tabular} & 230 & $\mathrm{H}, \mathrm{HL}, \mathrm{HB}, \mathrm{FL}$ & $171.12 / 159.43$ & \multicolumn{2}{|c|}{ Male - 25.86, Female - 22.67} & NM & NM \\
\hline 9 & $\begin{array}{c}\text { Uhrova P, Benus R et } \\
\left.\text { al10 }^{10} 2015\right) \text { - Slovak } \\
\text { adults } \\
\end{array}$ & 250 & H, HL, HB, FL, FB & NA & NA & NA & NA & NA \\
\hline 10 & $\begin{array}{c}\text { Rati Tandon et al }{ }^{17} \\
(2016)\end{array}$ & 497 & $\begin{array}{c}\text { H, HL, HB, FL, FB, } \\
\text { DL }\end{array}$ & $172.7 / 157.1$ & \multicolumn{2}{|c|}{ Male - 26.22, Female - 23.35} & \multicolumn{2}{|c|}{ Male - 9.95, female - 8.89} \\
\hline 11 & Dhaneria et al 18 & 500 & $\mathrm{H}, \mathrm{FL}, \mathrm{FB}$ & $166.43 / 160.91$ & \multicolumn{2}{|c|}{ Male - 24.71, female - 23.56} & \multicolumn{2}{|c|}{ Male -9.07 , female -8.68} \\
\hline 12 & $\begin{array}{c}\text { Kim W, Kim YM et al20 } \\
\text { (2018) }\end{array}$ & 5195 & H, HL, HB, FL, FB & NA & NA & NA & NA & NA \\
\hline
\end{tabular}

Table 6. Studies with Observations of Height and Foot Dimensions

H- Height, HL - Hand length, HB - Hand breadth, FL - Foot length, FB - Foot breadth, PL - Palm length, DL - Digit/ finger length, AS-Arm span, FAL - Forearm length, NM - Not measured NC - Not comparable, NA - Data not available. 


\begin{tabular}{|c|c|c|c|c|c|c|c|c|c|}
\hline $\begin{array}{l}\text { Sl. } \\
\text { No. }\end{array}$ & $\begin{array}{l}\text { Name of } \\
\text { Study }\end{array}$ & \multicolumn{5}{|c|}{ Correlation Coefficient ' $r$ ' } & \multicolumn{2}{|l|}{ Linear Regression Equation 'b' } & Conclusions \\
\hline 1 & $\begin{array}{c}\text { Oommen } \\
\text { et al }{ }^{1}\end{array}$ & \multicolumn{5}{|c|}{ Not calculated } & \multicolumn{2}{|l|}{ Not calculated } & $\begin{array}{c}\text { Conclusions not relevant to this } \\
\text { review study }\end{array}$ \\
\hline \multirow{2}{*}{2} & \multirow{2}{*}{$\begin{array}{l}\text { Danborno } \\
\text { and Elukpo }\end{array}$} & \multicolumn{5}{|c|}{$\begin{array}{c}\text { Male: } \mathrm{RHL}-0.53, \mathrm{LHL}-0.55, \mathrm{RFL}-0.58, \\
\text { LFL }-0.61\end{array}$} & \multicolumn{2}{|c|}{$\begin{array}{c}\text { Male: } \mathrm{Ht} .=61.036+(1.579 \times \mathrm{RHL})- \\
(1.003 \times \mathrm{LHL})+(3.524 \times \mathrm{RFL})+ \\
(0.0817 \times \mathrm{LFL})\end{array}$} & \multirow{2}{*}{$\begin{array}{l}\text { Height can be significant } \\
\text { predicted from hand and foot } \\
\text { length. }\end{array}$} \\
\hline & & \multicolumn{5}{|c|}{$\begin{array}{c}\text { Female: RHL - 0.43, LHL - 0.40, RFL- } \\
\text { 0.61, LFL- } 0.40\end{array}$} & \multicolumn{2}{|c|}{$\begin{array}{c}\text { Female: } \mathrm{Ht} .=71.965+(0.0276 \times \mathrm{RHL}) \\
-(2.410 \times \mathrm{LHL})+(0.268 \times \mathrm{RFL})+ \\
(1.787 \times \mathrm{LFL})\end{array}$} & \\
\hline \multirow{2}{*}{3} & \multirow{2}{*}{ Patel et $\mathrm{al}^{3}$} & \multicolumn{5}{|c|}{$\begin{array}{l}\text { Male: } 0.65 \text {; between height and foot } \\
\text { length }\end{array}$} & \multicolumn{2}{|l|}{ Male: Ht. $=75.45+3.64 \times$ FL } & \multirow{2}{*}{$\begin{array}{l}\text { Strong relationship between } \\
\text { height and foot length. }\end{array}$} \\
\hline & & \multicolumn{5}{|c|}{$\begin{array}{c}\text { Female: } 0.80 \text {; between height and foot } \\
\text { length }\end{array}$} & \multicolumn{2}{|l|}{ Female: Ht. $=75.41+3.43 \times$ FL } & \\
\hline 4 & $\mid \begin{array}{c}\text { Krishnan K } \\
\text { et } \mathrm{al}^{4}(2007)\end{array}$ & \multicolumn{5}{|c|}{$\begin{array}{c}\text { The correlation coefficients between } \\
\text { stature and all the measurements of } \\
\text { hands and feet were found to be positive } \\
\text { and statistically significant. }\end{array}$} & \multicolumn{2}{|l|}{ Date not available } & $\begin{array}{l}\text { Foot length provides highest } \\
\text { reliability and accuracy in } \\
\text { estimating stature of an } \\
\text { unknown individual. } \\
\end{array}$ \\
\hline 5 & $\begin{array}{l}\text { Rastogi } \\
\text { et } \mathrm{al}^{5}\end{array}$ & \multicolumn{5}{|c|}{\begin{tabular}{|} 
North Indian male -0.673 to 0.665 , North \\
Indian female: 0.740 to 0.732 ; South \\
Indian male -0.752 to 0.732 , South \\
Indian female -0.701 to 0.691
\end{tabular}} & \multicolumn{2}{|l|}{ Data not available } & $\begin{array}{l}\text { HL- } 2 \text { gives more accurate } \\
\text { results in stature estimation } \\
\text { than the HL-1. }\end{array}$ \\
\hline 6 & $\begin{array}{l}\text { Sen J } \\
\text { et } \mathrm{al}^{6}\end{array}$ & \multicolumn{5}{|c|}{$\begin{array}{c}\text { Stature, foot length and foot breadth are } \\
\text { positively and significantly correlated } \\
\text { with each other. The higher correlation } \\
\text { coefficient between stature and foot } \\
\text { length was seen than that between } \\
\text { stature and foot breadth. } \\
\end{array}$} & \multicolumn{2}{|l|}{ Data not available } & $\begin{array}{l}\text { Stature was strongly dependent } \\
\text { on foot length and foot breadth. }\end{array}$ \\
\hline 7 & $\begin{array}{c}\text { Kanchan } \\
\text { et } \text { al }^{7}(2008)\end{array}$ & \multicolumn{5}{|c|}{$\begin{array}{c}\text { The correlation coefficients between } \\
\text { stature and foot dimensions were found } \\
\text { to be positive and statistically highly } \\
\text { significant. The highest correlation } \\
\text { coefficient was observed between } \\
\text { stature and foot length in males and foot } \\
\text { breadth in females. }\end{array}$} & & & $\begin{array}{l}\text { Foot length provides the highest } \\
\text { reliability and accuracy in } \\
\text { estimating stature of an } \\
\text { unknown male and foot breadth } \\
\text { in a female. }\end{array}$ \\
\hline \multirow{4}{*}{8} & \multirow{4}{*}{$\begin{array}{l}\text { Chikhalkar } \\
\text { et al }{ }^{8}\end{array}$} & \multirow{4}{*}{\multicolumn{5}{|c|}{$\begin{array}{c}\text { HL }-0.5902, \text { HB- } 0.6004, \text { FL }-0.6102, \text { FB }- \\
0.4886\end{array}$}} & $\begin{array}{r}\mathrm{Y}=116.892872+2.665389 * \mathrm{X} \\
\text { Stature of individual, X: Hand-ler }\end{array}$ & & \multirow{4}{*}{$\begin{array}{l}\text { The study highlights that hand } \\
\text { length, hand width, forearm } \\
\text { length foot length \& foot width } \\
\text { can be reliably used for } \\
\text { estimation of stature in the } \\
\text { region of Mumbai. }\end{array}$} \\
\hline & & & & & & & $\begin{array}{r}\mathrm{Y}=113.561732+7.139216^{*} \mathrm{X} \\
\text { Stature of individual, } \mathrm{X}: \text { Hand-wi }\end{array}$ & & \\
\hline & & & & & & & $\begin{array}{r}\mathrm{Y}=79.72379+3.650632 * \mathrm{X}( \\
\text { Stature of individual, X: Foot-len }\end{array}$ & & \\
\hline & & & & & & & $\begin{array}{l}\mathrm{Y}=114.828119+5.906901 * \mathrm{X} \\
\text { Stature of individual, } \mathrm{X}: \text { Foot-wi }\end{array}$ & & \\
\hline 9 & $\begin{array}{c}\text { Krishan } \\
\text { et al }{ }^{9}\end{array}$ & \multicolumn{5}{|c|}{ Data not available } & \multicolumn{2}{|l|}{ Data not available } & $\begin{array}{l}\text { Correlation with sex was } \\
\text { studied. }\end{array}$ \\
\hline 10 & Patel, et al10 & \multicolumn{5}{|c|}{$\begin{array}{c}\mathrm{HL}-0.806, \mathrm{HB}-0.467, \mathrm{FL}-0.767, \mathrm{FB}- \\
0.665\end{array}$} & \multicolumn{2}{|l|}{$\begin{array}{c}\mathrm{Ht} .=59.52+5.9163 \times \mathrm{HL} \\
\mathrm{Ht} .=121.69+5.4188 \times \mathrm{HB} \\
\mathrm{Ht} .=70.37+3.8969 \times \mathrm{FL} \\
\mathrm{Ht} .=99.05+7.063 \times \mathrm{FB}\end{array}$} & $\begin{array}{c}\text { The highest correlation was } \\
\text { found between stature and Arm } \\
\text { span ( } \mathrm{r}=0.908) \text { followed by } \\
\text { hand length (0.806), followed } \\
\text { by foot length (0.767). }\end{array}$ \\
\hline 11 & $\begin{array}{l}\text { Ibegbu, } \\
\text { et al } 11\end{array}$ & & ta not $c$ & ompar & able & & Data not comparable & & Data not comparable \\
\hline 12 & $\begin{array}{l}\text { Mohite, } \\
\text { et al12 }\end{array}$ & $\mathrm{HL}-0.3$ & $38, \mathrm{HB}-$ & $-0.74, \mathrm{I}$ & $F L-0.0$ & & $\begin{array}{c}\text { Height }=65.60+0.54 \times \text { Hand Len } \\
\text { Height }=104.03+0.76 \times \text { Han } \\
\text { Breadth Height }=64.81+0.42 \times \\
\text { Length }\end{array}$ & & $\begin{array}{c}\text { Foot length is the most reliable } \\
\text { predictor for estimating the } \\
\text { stature of an Individual as } \\
\text { observed in our study. }\end{array}$ \\
\hline 13 & $\begin{array}{c}\text { Bodorikova } \\
\text { and } \\
\text { Nescakova } 13\end{array}$ & $\begin{array}{r}\text { The highest } \\
\text { found for foo } \\
\text { well a }\end{array}$ & $\begin{array}{l}\text { correla } \\
\text { t length } \\
\text { in in fem }\end{array}$ & $\begin{array}{l}\text { ation co } \\
\text { in mal } \\
\text { nales }(r\end{array}$ & $\begin{array}{l}\text { efficien } \\
\text { les }(\mathrm{r}=0 \\
=0.63)\end{array}$ & $\begin{array}{l}\text { at was } \\
\text {.71) as }\end{array}$ & Data not available & & $\begin{array}{c}\text { There was a positive and } \\
\text { statistically significant } \\
\text { correlation between stature and } \\
\text { all measurements in both sexes } \\
(\mathrm{p}<0.01) .\end{array}$ \\
\hline & & Correlation & & SIM & & NIM & $\begin{array}{c}\mathrm{S}=113.08+6.27 * \text { Left hand } \\
\text { breadth }\end{array}$ & & \\
\hline 14 & Kavyashree & $\underset{\text { ' } r \text { ' }}{\text { Coefficent }}$ & SIF & SIIVI & NIF & NIIV & $\begin{array}{c}\mathrm{S}=122.49+2.07 * \text { Left hand } \\
\text { length }\end{array}$ & NF & $\begin{array}{l}\text { Study shows a positive } \\
\text { correlation of stature with hand }\end{array}$ \\
\hline & & RHL & 0.412 & 0.414 & 0.367 & 0.541 & $\begin{array}{c}\mathrm{S}=103.89+7.52 * \text { Right hand } \\
\text { breadth }\end{array}$ & & measurements. \\
\hline & & & & & & & $\mathrm{S}=127.15+1.8 *$ Right hand & & \\
\hline
\end{tabular}




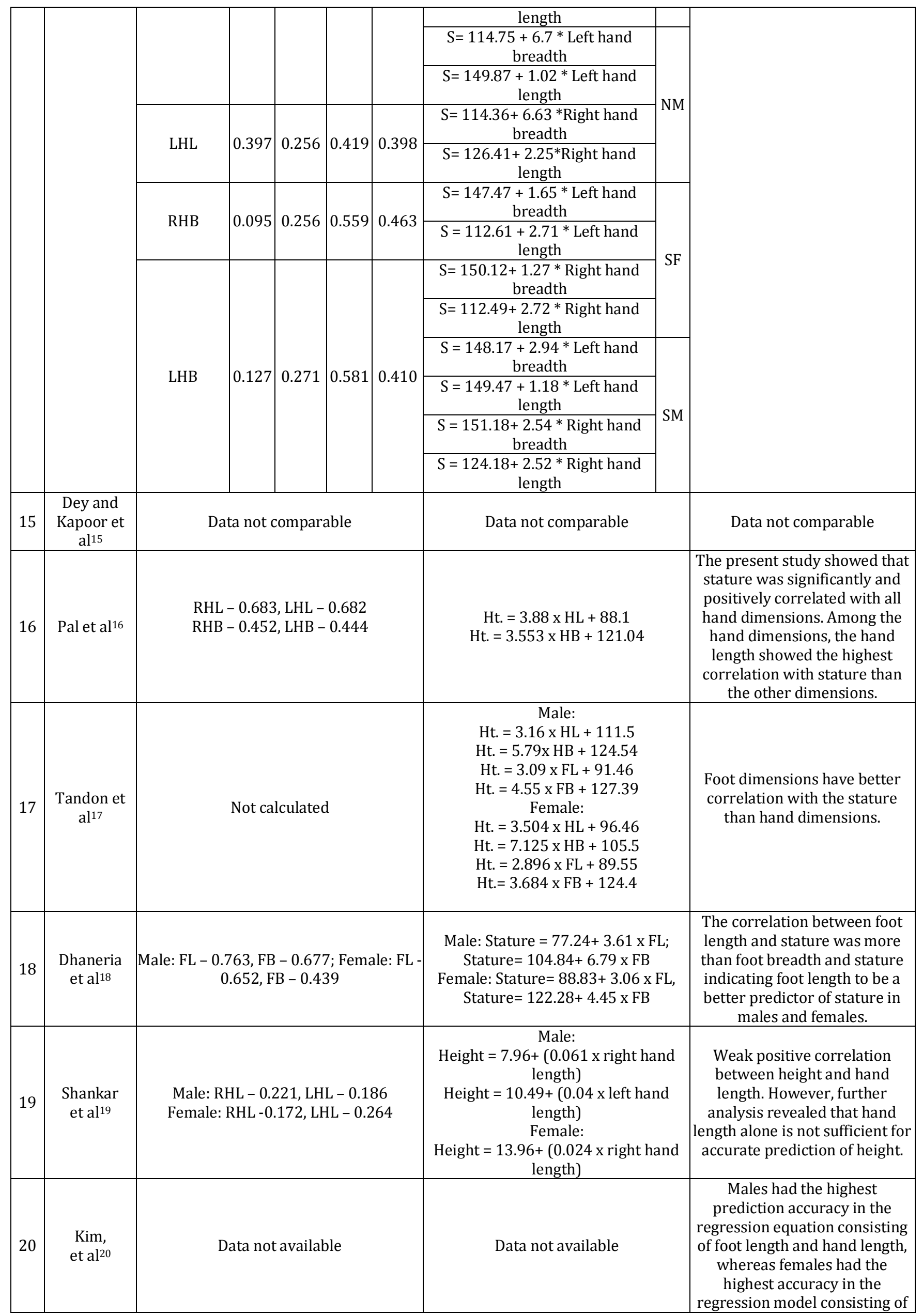




\begin{tabular}{|c|c|c|c|c|}
\hline & & & & foot length and hand width. \\
\hline 21 & $\begin{array}{c}\text { Samoon } \\
\text { et } \mathrm{al}^{21}\end{array}$ & RHL - 0.787941, LHL - 0.777245 & Not calculated & $\begin{array}{l}\text { In our present study we } \\
\text { correlated the mean hand } \\
\text { lengths of both sides with the } \\
\text { stature and found a statistically } \\
\text { significant correlation. }\end{array}$ \\
\hline 22 & $\begin{array}{l}\text { Ibrahim } \\
\text { et al22 }\end{array}$ & $\begin{array}{c}\text { Male: } \\
\text { RHL -0.665, LHL-0.388, } \\
\text { RHB- 0.560, LHB-0.496; } \\
\text { Female: } \\
\text { RHL- 0.760, LHL-0.612, } \\
\text { RHB-0.490, LHB-0.436 }\end{array}$ & $\begin{array}{c}\text { Male: } \\
\mathrm{S}=92.468+4.132 \times \mathrm{HL}, \\
\mathrm{S}=104.334+4.572 \times \mathrm{HB} \\
\text { Female: } \\
\mathrm{S}=88.623+4.567 \mathrm{HL} \\
\mathrm{S}=138.022+4.563 \mathrm{HB}\end{array}$ & $\begin{array}{c}\text { The current study cleared a } \\
\text { significant positive correlation } \\
\text { between the stature and the } \\
\text { measured hand dimensions. } \\
\text { This correlation is stronger in } \\
\text { hand length than other } \\
\text { parameters. }\end{array}$ \\
\hline \multicolumn{5}{|c|}{ Table 7. Studies with Observations of Correlation Coefficient and Equation } \\
\hline
\end{tabular}

\section{DISCUSSION}

Correlation of Height and Dimensions of Hand and Foot Patel et al, ${ }^{3}$ Krishnan K et al ${ }^{4}$ (2007), Sen J et al, ${ }^{6}$ Kanchan et $\mathrm{al}^{7}$ (2008), Dhaneria et al ${ }^{18}$ studied correlation of height with foot dimensions and observed statistically significant correlation between them. Chikhalkar et $\mathrm{al}^{8}$ Patel et al,10 Mohite et $\mathrm{al}^{12}$ studied height and dimensions of hand and foot and observed statistically significant correlation between them. Kavyashree et al,14 Pal et al,16 Tandon et al,17 Shankar et al,19 Samoon et $\mathrm{al}^{21}$ studied height with dimensions of hand and observed statistically significant correlation between them. Dhaneria et al,18 Tandon et al,17 Patel et al,10 Mohite et al,12 Kanchan et $\mathrm{al}^{7}$ (2008), Krishnan K et al ${ }^{4}$ (2007) in their studies stated that dimensions of foot especially foot length is a better predictor of stature in males and females.

\section{Comparison of Height in Indian Population}

Kavyashree et al, ${ }^{14}$ Shankar et al, $^{19}$ Oommen et al ${ }^{1}$ studied south Indian population while almost all the remaining authors (table 1) studied north Indian subjects. Mean height in north Indian population ranged from 172.7 to $166.43 \mathrm{~cm}$ in male and from 160.91 to $156.14 \mathrm{~cm}$ in female. Mean height in south Indian population was $171.73 \mathrm{~cm}$ in male and was $159.25 \mathrm{~cm}$ in female. So, it can be said that mean height is more in north Indian population compared to their south Indian population. (Table 5).

\section{Comparison of Hand Dimensions in Indian Population}

Mean hand length in north Indian population on right side ranged from 19.3 to $18.21 \mathrm{~cm}$ and on left side from 19.0 to $18.35 \mathrm{~cm}$ in male. Mean hand length in north Indian population on right side ranged from 18.81 to $17.3 \mathrm{~cm}$ and on left side from 18.82 to $16.5 \mathrm{~cm}$ in female.

Mean hand length in south Indian population on right side was $18.21 \mathrm{~cm}$ and on left side was $18.31 \mathrm{~cm}$ in male. Mean hand length in south Indian population on right side was $18.81 \mathrm{~cm}$ and on left side was $18.82 \mathrm{~cm}$ in female.

Mean hand width in north Indian population on right side was $8.3 \mathrm{~cm}$ and on left side was $8.18 \mathrm{~cm}$ in male. Mean hand width in north Indian population on right side was $7.57 \mathrm{~cm}$ and on left side was $7.45 \mathrm{~cm}$ in female.

Mean hand width in south Indian population was $8.24 \mathrm{~cm}$ in male. Mean hand width in south Indian population was $8.00 \mathrm{~cm}$ in female.

Hence it can be stated that dimensions of hand are more in north Indian males compared to south Indian males. Hand width dimensions are higher in south Indian females compared to their north Indian females. (Table 5)

\section{Comparison of Foot Dimensions in Indian Population}

Mean foot length in north Indian population on right side ranged from 26.22 to $24.44 \mathrm{~cm}$ and on left side from 26.22 to $24.44 \mathrm{~cm}$ in male. Mean foot length in north Indian population on right side ranged from 23.75 to $22.34 \mathrm{~cm}$ and on left side from 23.68 to $22.34 \mathrm{~cm}$ in female.

Only three studies namely Kavyashree et al ${ }^{14}$, Shankar et $\mathrm{al}^{19}$ and Oommen et $\mathrm{al}^{1}$ were conducted in south Indian

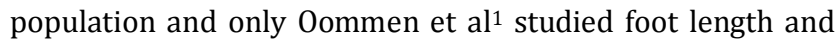
height. She stated that mean foot length in south Indian population on right side was $26.21 \mathrm{~cm}$ and on left side was $26.0 \mathrm{~cm}$ in male. Mean foot length in south Indian population on right side was $23.75 \mathrm{~cm}$ and on left side was $23.68 \mathrm{~cm}$ in female.

Mean foot width in north Indian population ranged from 9.07 to $9.95 \mathrm{~cm}$ in male whereas it ranged from 8.68 to 8.89 $\mathrm{cm}$ in female. No data was available for foot width in south Indian population.

Hence it can be commented that mean foot length was observed higher in north Indian males compared to south Indian males. But no significant difference was observed between north and south Indian females. Whereas mean foot width couldn't be compared between north and south Indian population.

\section{CONCLUSIONS}

1. Significant positive correlation was observed between human stature and hand and foot dimensions.

2. Foot length was stated as a better predictor of stature compared to other dimensions.

3. Human stature of north Indian population is higher than that of south Indian population.

4. Hand and foot dimensions are higher in north Indian males than in south Indian males.

5. It would be unwise to use the same equations for stature estimation for different Indian populations.

6. The data collected should be useful for anthropologists and forensic experts.

\section{REFERENCES}

[1] Oommen A, Mainker A, Oommen T. A study of the correlation between hand length and foot length in humans. J Anat Soc India 2005;54(2):55-7. 
[2] Danborno B, Elukpo A. Sexual dimorphism in hand and foot length, indices, stature-ratio and relationship to height in Nigerians. The Internet Journal of Forensic Science 2007;3(1):1-5.

[3] Patel SM, Shah GV, Patel SV. Estimation of height from measurements of foot length in Gujarat region. J Anatomy Soc India 2007;56(1):25-7.

[4] Krishan K, Sharma A. Estimation of stature from dimensions of hands and feet in a North Indian population. J Forensic Leg Med 2007;14(6):327-32.

[5] Rastogi P, Nagesh KR, Yoganarasimha K. Estimation of stature from hand dimensions of North \& South Indians. Legal Medicine (Tokyo) 2008;10(4):185-9.

[6] Sen J, Ghosh S. Estimation of stature from foot length and foot breadth among the Rajbanshi: an indigenous population of North Bengal. Forensic Sci Int 2008;181(1-3):55.e1-6.

[7] Kanchan T, Meneses RG, Moudgil R, et al. Stature estimation from foot dimensions. Forensic Sci Int 2008;179(2-3):241.e1-5.

[8] Chikhalkar BG, Mangaonkar AA, Nanandkar SD, et al. Estimation of stature from measurements of long bones, hand and foot dimensions. J Indian Acad Forensic Med 2009;32(4):329-30.

[9] Krishan K, Kanchan T, Sharma A. Sex determination from hand and foot dimensions in a North Indian population. J Forensic Sci 2011;56(2):453-9.

[10] Patel PN, Tanna JA, Kalele SD. Correlation between hand length and various anthropometric parameters. International Journal of Medical Toxicology and Forensic Medicine 2012;2(2):61-3.

[11] Ibegbu AO, David ET, Hamman WO, et al. Association of hand length with height in Nigerian school children. Journal of Biology and Life Science 2013;4(2):83-94.

[12] Mohite PM, Keche AS, Mohite DP, et al. Correlation of the dimensions of hand \& feet with stature of an individual: a study on central Indian adults. J Indian Acad Forensic Med 2015;37(2):160-4.
[13] Uhrova P, Benus R, Masnicova S, et al. Estimation of stature using hand and foot dimensions in Slovak adults. J Leg Med (Tokyo) 2015;17(2):92-7.

[14] Kavyashree AN, Bindurani MK, Asha KR. Determination of stature from hand dimensions in Indian population. Journal of International Medicine and Dentistry 2015;2(3):209-14.

[15] Dey S, Kapoor AK. Hand length and hand breadth: a study of correlation statistics among human population. International Journal of Science and Research 2015;4(4):148-50.

[16] Pal A, De S, Sengupta P, et al. Estimation of stature from hand dimensions in Bengalee population, West Bengal, India. Egyptian Journal of Forensic Sciences 2016;6:90-8.

[17] Tandon R, Yunus SM, Faruqi NA, et al. Measurements of hand and foot - a predictor of stature in adult human population of Uttar Pradesh. International Journal of Anatomy, Radiology and Surgery 2016;5(1):12-5.

[18] Dhaneria V, Shrivastava M, Mathur RK, et al. Estimation of height from measurement of foot breadth and foot length in adult population of Rajasthan. Indian Journal of Clinical Anatomy and Physiology 2016;3(1):78-82.

[19] Shankar GS, Shankar VV, Radhika K, et al. Correlation of human height with hand length in Indian individuals. Int J Anat Res 2017;5(4.1):4478-81.

[20] Kim W, Kim YM, Yun MH. Estimation of stature from hand and foot dimensions in a Korean population. J Forensic Leg Med 2018;55:87-92.

[21] Samoon S, Itoo MS, Shahdad S, et al. Correlation of stature and hand length in adult Kashmiri population. International Journal of Contemporary Medical Research 2018;5(3):C1-C2.

[22] Ibrahim MA, Khalifa AM, Hassan HA, et al. Estimation of stature from hand dimensions in North Saudi population, medicolegal view. Saudi J Forensic Med \& Sci 2018;1(1):19-27. 